\title{
Clinical Feature and Bowel Ultrasound in Crohn's Disease - Does Additional Information from Magnetic Resonance Imaging Affect Therapeutic Approach and When Does Extended Diagnostic Investigation Make Sense?
}

\author{
Christiane Girlich $^{\mathrm{a}}$ Claudia Ott $^{\mathrm{a}} \quad$ Ulrike Strauch ${ }^{\mathrm{a}}$ Doris Schacherer ${ }^{\mathrm{a}}$ \\ Florian Obermeier $^{\mathrm{a}} \quad$ Ernst Michael Jung ${ }^{\mathrm{b}}$ Jürgen Schölmerich ${ }^{\mathrm{a}}$ \\ Andreas Georg Schreyer ${ }^{b} \quad$ Frank Klebl ${ }^{a}$ \\ Departments of ${ }^{a}$ Internal Medicine I, and ${ }^{b}$ Radiology, University of Regensburg, Regensburg, Germany
}

\section{Key Words}

Crohn's disease $\cdot$ MR enterography $\cdot$ Ultrasound .

Therapeutic approach

\begin{abstract}
Background/Aims: Some suggest MRI to be superior to ultrasound in Crohn's disease. We analyzed how often MR enterography (MRE) following a routine ultrasound leads to a change in therapeutic decision. Material and Methods: We retrospectively evaluated 47 patients with Crohn's disease undergoing routine ultrasound examination. Actual medical history, complete blood count, C-reactive protein (CRP), and sonographic findings were assessed independently by two specialists who retrospectively provided a therapeutic proposal. Additionally, all patients received MRE. Thereafter, the specialists had to provide a new therapeutic concept regarding all the available information. Results: Evaluation of the rectum was not successful by ultrasound, but MRE gave good results. Only 1 of 7 abscesses was identified sonographically. Three of the abscesses missed at sonography were localized in the perirectal/perianal region. MRE detected more inflamed bowel segments, but ultrasound assess-
\end{abstract}

ment of anatomically fixed bowel parts showed good recognition by MRE. With increasing CRP values, we found more positive results of ultrasound and MRE. Therapeutic change was suggested in only 18 patients. Conclusions: Ultrasound should be performed by an experienced examiner, and a proctological examination should be added. MRE is justified in cases of discrepancy between clinical findings and the results of diagnostic ultrasound and, moreover, if Crohn's lesions are suspected at sites proximal to the terminal or neoterminal ileum.

Copyright $\odot 2010$ S. Karger AG, Basel

\section{Introduction}

Crohn's disease is a chronic relapsing and remitting disorder, presenting as a granulomatous, transmural and discontinuous inflammation affecting the wall of the gastrointestinal tract. Although the inflammation can occur everywhere from the mouth to the anus, it particularly involves the terminal ileum and the proximal colon. Recently, magnetic resonance enterography (MRE) as well as computed tomography enterography (CTE) is be-

\section{KARGER}

Fax +4161306 1234 E-Mail karger@karger.ch www.karger.com
(C) 2010 S. Karger AG, Basel

$0012-2823 / 11 / 0832-0018 \$ 38.00 / 0$

Accessible online at:

www.karger.com/dig
Dr. med. Christiane Girlich

Department of Internal Medicine I, University Hospital of Regensburg

DE-93042 Regensburg (Germany)

Tel. +49941944 7003, Fax +499419447004

E-Mail christiane.girlich@ klinik.uni-regensburg.de 
ing accepted more and more as the first-line modality for the evaluation of suspected inflammatory bowel disease [1-5]. Ultrasound as a widely available, non-radiation examination can be performed for assessment of bowel wall abnormalities and complications of Crohn's disease such as fistulas and abscesses [6, 7]. Due to increased vascularization in inflammation, an enhanced Doppler signal can be found; however, these changes in wall vascularity visualized by Doppler techniques $[4,8,9]$ show no clear correlation with clinical or biological scores $[6,10]$. Some suggest MRE to be superior to ultrasound, particularly regarding the localization of affected bowel segments and the detection of fistulas [11]. Other studies showed a good comparability between the results of MR enterography and ultrasound [12-14].

It has to be kept in mind that in all these studies ultrasound was performed by a very experienced examiner being engaged only with the object of the study and not being subjected to any time pressure regarding the examination. The aim of our study was to analyze in how many cases a MRE compared with a routine ultrasound done in an interdisciplinary center of clinical ultrasound (3 examiners, 60-80 ultrasound examinations a day) during normal working time without any special conditions leads to a change in a therapeutic decision. In other words, how good is ultrasound under 'real working conditions' and what are the implications?

\section{Material and Methods}

We retrospectively evaluated findings of MRE and ultrasound in 47 in- and outpatients (21 males, 26 females, mean age $31.8 \pm$ 11.4 years) with previously diagnosed Crohn's disease who underwent ultrasound as well as MRE. Routine B-scan and Doppler ultrasound were performed with a linear $7.5-\mathrm{MHz}$ transducer (Elegra, Siemens, Germany; Logiq 9, General Electric, USA) carried out by one of three examiners in our interdisciplinary center of clinical ultrasound (60-80 ultrasound examinations a day) during working time $(08.00$ until $17.00 \mathrm{~h})$ without any special conditions. Bowel wall thickening $(>3 \mathrm{~mm})$ with hyperperfusion to a greater or lesser extent suggested affection by Crohn's disease. Additionally, all patients received MRE in the 'dark lumen' technique as described elsewhere [15]. Image evaluation was performed by two experienced radiologists in consensus by means of bowel wall thickening and increased contrast uptake of the bowel wall. The examiners were blinded to the results of the other imaging modality. Delay between the two examinations was $5.8 \pm 7.0$ days in all 47 patients. Thereafter, actual medical history, complete blood count, C-reactive protein (CRP) and sonographic Bscan findings were independently assessed by two board-certified physicians with extended experience in the diagnosis and therapy of Crohn's disease. No relevant therapeutic actions, such as administration of high dose steroids or anti-TNF agents, were taken between the performances of ultrasound and MRE, respectively. Based on these examinations, the two specialists retrospectively provided a therapeutic concept. Thereafter, the physicians were additionally given the results of the MRE, and they had to provide a new proposal of therapy regarding all the information available (current medical history, complete blood count, CRP (normal value $<5 \mathrm{mg} / \mathrm{l}$ ), results of B-scan ultrasound with Doppler and results of MRE). Considering the results of MRE, possible criteria for a change in therapeutic proposal were a change in the feature 'localization' of the Montreal classification (L1 ileal, L2 colonic, L3 ileocolonic, L4 isolated upper gastrointestinal tract, L4 + L1/ L2/L3 upper and L1/L2/L3) and a newly diagnosed abscess. Regarding these criteria, one has to keep in mind that - due to information about clinical presentation and preexisting therapy not every additional information from MRE leads to another therapeutic proposal. Since neither a transrectal ultrasound nor a magnetic resonance imaging of the pelvis - each the method of choice for the detection of pararectal fistulas - were performed in our study, we did not regard pararectal fistulas. The ethics committee at the University of Regensburg approved the study.

\section{Results}

Ultrasound Findings and Their Correlation with the Results of the MR Examinations

Sonographically, the most frequent affected bowel segments were found in the ileum and the terminal ileum - representing the preferential site of Crohn's disease. 17 of 19 findings in the ileum were confirmed by MRE. The right and the left colon were the second most frequent affected sites, and MRE could reproduce all sonographic findings in these segments. The only case of inflammation of the jejunum detected by ultrasound was not confirmed by MRE. Ultrasound did not identify any inflammation of the rectum in the 47 patients. In one case, sonographic findings showed an abscess (fig. 1).

\section{Correlation of MRE Assessment with Ultrasound \\ Findings of the Corresponding Sites}

Affection of the ileum was seen 31 times by MRE; however, only 17 cases of them could also be identified by ultrasound. Ultrasound could not detect any of the jejunal segments affected according to MRE (0/3), furthermore, no rectal (0/7) affection. Seven abscesses were diagnosed by MRE, but only one abscess was seen by ultrasound (fig. 2). Six patients suffering from abscesses had clearly elevated inflammation markers (CRP $>50 \mathrm{mg} / \mathrm{l}$ ), but only 1 of them presented with fever. The seventh patient had no fever, but showed a CRP value of only 30.69 $\mathrm{mg} / \mathrm{l}$; however, the abscess was only about $1 \mathrm{~cm}$ in size (see below). 


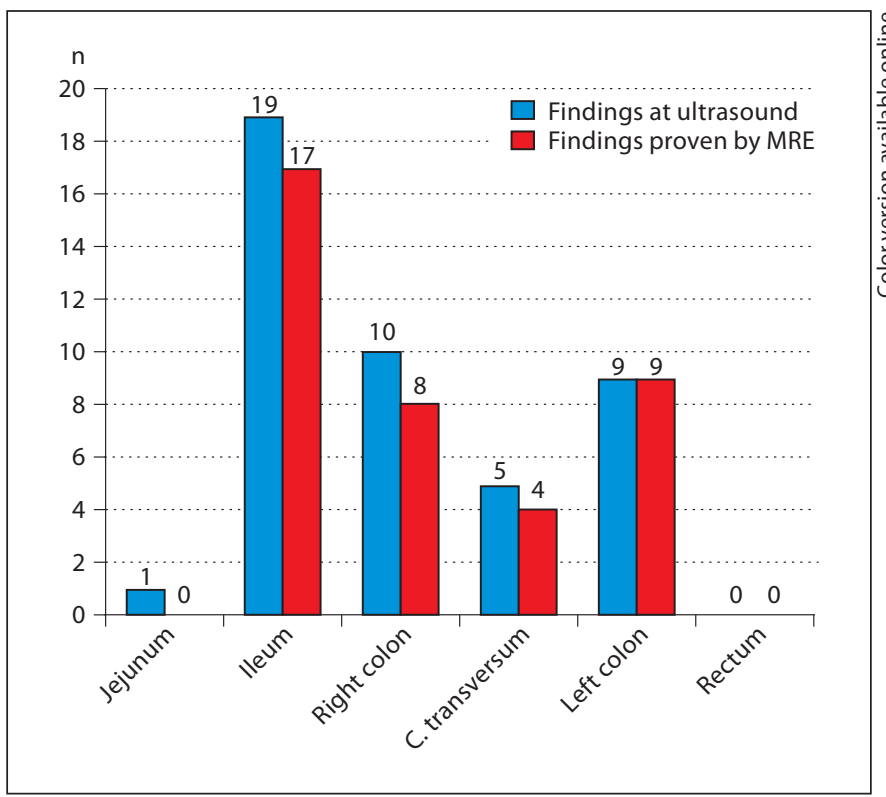

Fig. 1. Ultrasound findings and their recognition by MRE.

Table 1. Change of L classification according to the Montreal classification

\begin{tabular}{ll}
\hline $\begin{array}{l}\text { Classification by } \\
\text { means of ultrasound }\end{array}$ & Change as a consequence of MRE results \\
\hline L1 & L3 in 6 cases \\
L4 + L1 in 1 case \\
\hline L2 & L1 in 1 case \\
& L3 in 2 cases \\
L4 + L3 in 1 case
\end{tabular}

$\mathrm{L}=$ Localization.

Correlation of Imaging Results with CRP Levels

With increasing CRP values, we observed an increasing number of positive ultrasounds and positive MREs. Two patients with a CRP value $>70 \mathrm{mg} / \mathrm{l}$ and no evidence of a manifestation of Crohn's disease at MRE and bowel ultrasound suffered from pancreatitis while taking azathioprine, and urosepsis with underlying nephrolithiasis,

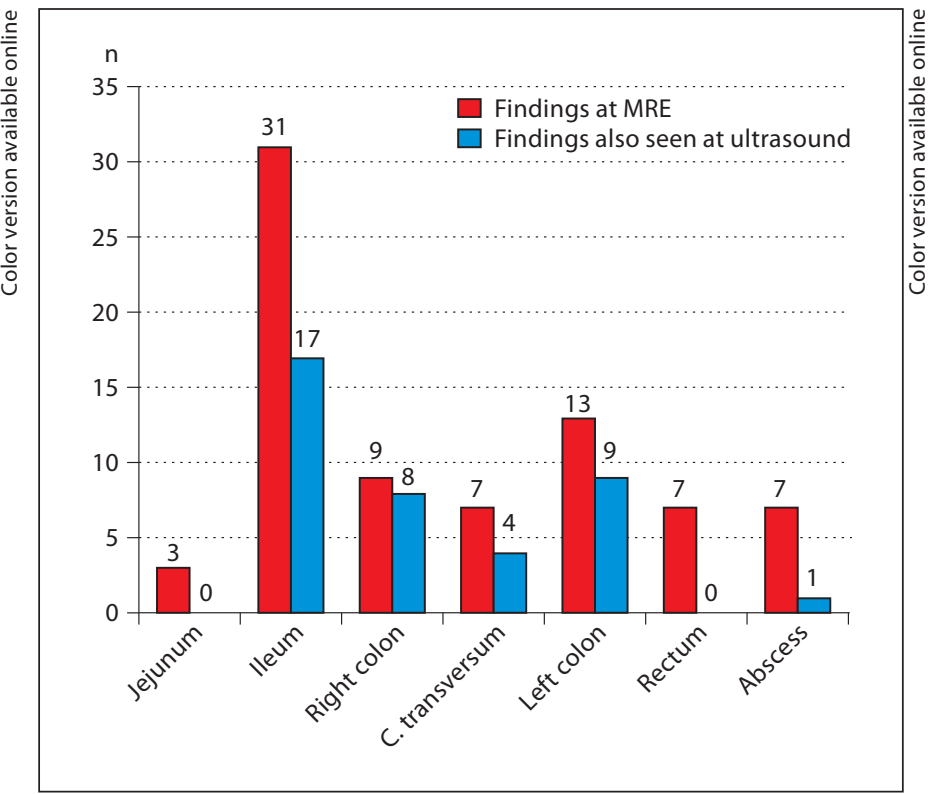

Fig. 2. MRE and ultrasound findings of the corresponding sites.

respectively. The third patient with normal ultrasound and CRP $>70 \mathrm{mg} / \mathrm{l}$ showed an ileitis and an abscess on MRE.

In contrast, there were 6 patients with unremarkable CRP values, but positive findings in MR examinations. These patients were treated with high-dose systemic corticosteroids $(\mathrm{n}=2)$, budesonide $(\mathrm{n}=1)$, azathioprine plus budesonide $(\mathrm{n}=1)$ or azathioprine plus steroids $(\mathrm{n}=1)$, and certolizumab $(\mathrm{n}=1)$, respectively (fig. 3$)$. No abscesses were detected with CRP levels below $20 \mathrm{mg} / \mathrm{l}$; the only 2 patients with CRP $<70 \mathrm{mg} / \mathrm{dl}$ and an abscess had CRP values of $58.53 \mathrm{mg} / \mathrm{l}$ (with methotrexate) and 30.69 $\mathrm{mg} / \mathrm{l}$ (without immunosuppressive therapy), respectively (fig. 4). However, the latter abscess was only about $1 \mathrm{~cm}$ in size. Three of the abscesses missed by sonography were localized in the pararectal or the perianal region, and one further abscess was seen to be only about $1 \mathrm{~cm}$ in size at MRI.

\section{Change of Known Bowel Involvement Pattern as a Consequence of MRE Findings}

Since therapeutic implications may arise from a change in knowledge of the pattern of bowel involvement, we analyzed this in our patients. In 24 cases, additional information from MR examinations resulted in a relevant change in the knowledge of affected bowel segments followed by a change of placement according to the Mon- 


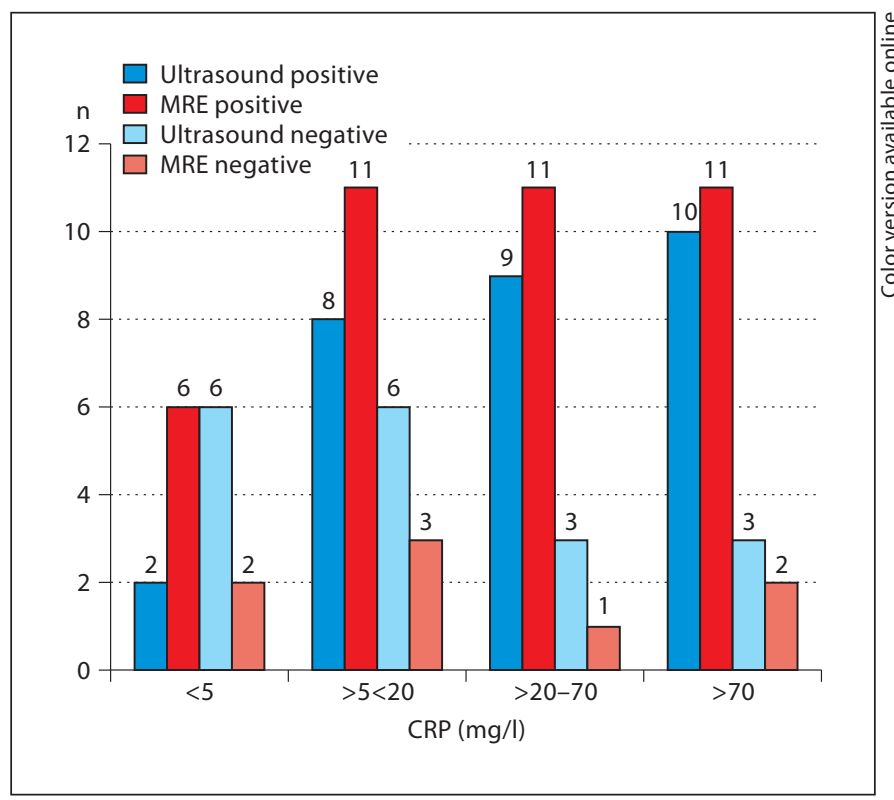

Fig. 3. Imaging results and CRP levels.

treal classification (table 1). In 7 cases, rectal involvement was not detected by transabdominal ultrasound but by MRE.

\section{Therapeutic Implications Arising from $M R$ \\ Examinations}

The initial interobserver variability was calculated. There was an observed interobserver agreement of $89 \%$ with $\kappa=0.76$ representing a substantial agreement between the specialists. The specialists discussed the 5 cases in which their assessment differed and reached agreement regarding their therapeutic proposals. Thus, in 18 of the total 47 patients (38\%), a change in therapeutic strategy was necessary based on the findings of MR examinations. Ultrasound was negative concerning Crohn's lesions 11 times, despite clinical findings suggestive of activity (e.g. pain, diarrhea), but MRE demonstrated bowel inflammation. An abscess found by MRE led to another therapeutic approach 4 times, and MRE diagnosed a more extended bowel involvement with Crohn's disease affecting either more or other bowel segments than ultrasound, causing a change in treatment strategy 3 times.

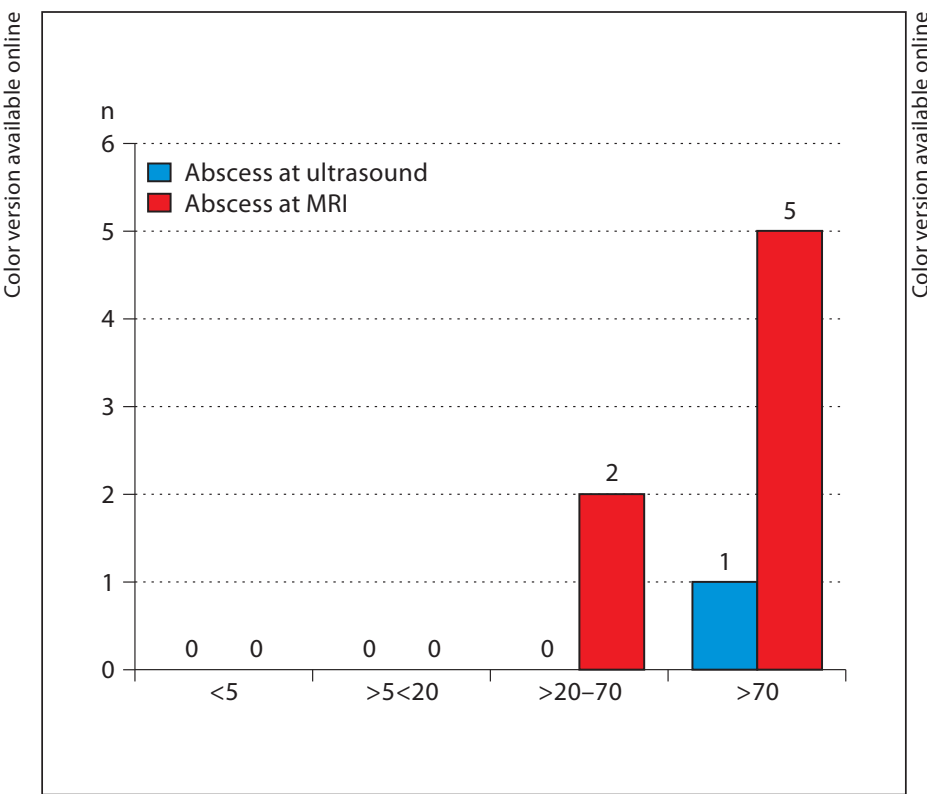

Fig. 4. Abscesses and CRP levels.

\section{Discussion}

Due to its characteristic as a chronic remitting and relapsing disease, patients with Crohn's disease again and again suffer from diarrhea and abdominal pain. These complaints are not always caused by bowel inflammation, but can also be based on Crohn's disease-associated affections such as (drug-induced) pancreatitis (azathioprine) or other non-Crohn's disease-related illnesses such as infectious gastroenteritis, nontropical sprue, urinary tract infection, gynecological disorders, and many more. Thus, besides clinical examinations and laboratory tests, patients with Crohn's disease often have to undergo several imaging studies in order to find the most suitable therapeutic approach. Ultrasound and MRE are the diagnostic imaging techniques of choice in the mostly young Crohn's disease patients since they are not associated with the exposure to irradiation, particularly as they are noninvasive.

Bowel wall ultrasound is an elegant method for clinical follow-up in patients with Crohn's disease. But it is known to have some limitations; thus, Schober et al. [16] recommend that ultrasound should be supplemented in both the primary diagnosis and follow-up with methods such as endoscopy and complementary imaging.

The reported ranges of ultrasound sensitivity and specificity for the diagnosis of Crohn's disease are 75-94 
and $67-100 \%$, respectively [17]; the values of MRI sensitivity and specificity for the diagnosis of Crohn's diseaserelated lesions range from 78 to $100 \%$ for both $[18,19]$.

A number of studies have been done comparing ultrasound and MRE with divergent results. Some studies showed a good comparability between the results of MRE and ultrasound, in some ultrasound was even superior $[12-14,20]$. Others suggested that MRE was superior to ultrasound, particularly regarding the localization of affected bowel segments and the detection of fistulas [11].

Similar to the results of Pascu et al. [13], evaluation of the rectum was not successful using ultrasound, whereas assessment by MRE was uncomplicated. Moreover, in our study, MRE detected far more inflamed bowel segments than did ultrasound. However, ultrasound assessment of the anatomically fixed bowel parts such as the terminal ileum and left colon showed good recognition by MRE, suggesting a good reliability of sonographically detected pathological findings of these bowel regions. Similar results were presented by Potthast et al. [11] who showed bowel wall ultrasound to have a high sensitivity $(85 \%)$ for recognizing involvement of the terminal or neoterminal ileum and a significantly lower sensitivity (50\%) for the evaluation of more proximal bowel segments. They attributed their findings to the fact that topographic orientation proximal to the terminal or neoterminal ileum is difficult with ultrasound.

We found an increasing number of positive ultrasounds and MR examinations with increasing CRP values. On the other hand, there were 2 patients who complained of abdominal pain with a CRP value $>70 \mathrm{mg} / \mathrm{l}$ who had no evidence of manifestation of Crohn's disease, but suffered from other diseases (pancreatitis and urosepsis based on nephrolithiasis). Also, there were 6 patients with unremarkable CRP values, but positive findings in MRE. All of these patients were treated with immunosuppressive drugs. Thus, we can conclude that CRP may provide an indication of an acute episode of Crohn's disease, but other causes have to be considered. Furthermore, patients on immunosuppressive drugs can suffer from an acute episode of Crohn's disease or abscess despite normal CRP values.

In our collective, we only found seven abscesses at MRE, and only one of them could be identified at ultrasound. Three of the six abscesses which were not detected by sonography were found in the perirectal/perianal region. Obviously, this region is difficult to assess reliably by transabdominal sonography; however, a transrectal sonography might have captured these three perirectal/ perianal abscesses. A further abscess was only about $1 \mathrm{~cm}$ in size. Hence, the markedly good specificity (92-94\%) and sensitivity (87-100\%) of ultrasound in the detection of abscesses reported by Potthast et al. [11], Maconi et al. [21] and Gasche et al. [22] can hardly refer to pararectal abscesses. In about half $(n=24)$ of our patients, a change in the knowledge of the involvement pattern was evident after MRE, this led to a change in therapeutic strategy as suggested by the specialists in only 16 of these patients. Overall, in 18 patients, the specialists provided a therapeutic change, the reason being - besides the above-mentioned change of the involvement pattern - the detection of abscesses in 4 cases (in 2 patients double finding of involvement pattern and abscess). However, MRE revealed rectal involvement in 7 patients, none of which were identified by ultrasound. The rectal involvement led to a change in the Montreal classification in 4 patients and to a change of therapeutic strategy proposed by the specialists in 3 patients. Thus, if transabdominal ultrasound would have been supplemented by proctoscopy, additional information from MRE concerning the involvement pattern (Montreal classification) could be assumed in only 22 patients, and a change of therapeutic proposal due to this additional information would have been made in only 14 patients. Moreover, if additional transrectal sonography would have been performed, the three perirectal/perianal abscesses could have been identified.

A limitation of our study is the lack of a reference standard and, hence, the validation of the ultrasound and MRE findings, respectively. Conventional enteroclysis and endoscopy represent the Goldstandard for diagnosis and evaluation of Crohn's disease concerning intraluminal changes; however, assessment of the transmural or extramural extent or extraintestinal complications is limited. Laparotomy as reference standard for extraluminal manifestations was not performed. Further studies comparing the capabilities of bowel wall ultrasound and MRE by using a reference standard will be required to confirm the findings of the present study.

Based on our results, we suggest - similar to Potthast et al. [11] - the following procedure in the assessment of bowel involvement in Crohn's disease: ultrasound should be performed by an experienced examiner, but in any case, a proctological examination should be added. MRE is justified in cases of discrepancy between clinical findings and/or laboratory tests and the results of diagnostic ultrasound. Magnetic resonance imaging is also useful in the diagnosis of intra-abdominal abscesses. Finally, MRE should be performed if Crohn's lesions are suspected at sites proximal to the terminal or neoterminal ileum. 


\section{References}

-1 Schreyer AG, Geissler A, Albrich H, Schölmerich J, Feuerbach S, Rogler G, Völk M, Herfarth H: Abdominal MRI after enteroclysis or with oral contrast in patients with suspected or proven Crohn's disease. Clin Gastroenterol Hepatol 2004;2:491-497.

$\checkmark 2$ Bernstein CN, Greenberg H, Boult I, Chubey S, Leblanc C, Ryner L: A prospective comparison study of MRI versus small bowel follow-through in recurrent Crohn's disease. Am J Gastroenterol 2005;100:2493-2502.

\3 Frokjaer JB, Larsen E, Steffensen E, Nielsen AH, Drewes AM: Magnetic resonance imaging of the small bowel in Crohn's disease. Scand J Gastroenterol 2005;40:832-842.

4 Rieber A, Wruk D, Potthast S, Nüssle K, Reinshagen M, Adler G, Brambs HJ: Diagnostic imaging in Crohn's disease: comparison of magnetic resonance imaging and conventional imaging methods. Int J Colorectal Dis 2000;15:176-181.

5 Paulsen SR, Huprich JE, Fletcher JG, Booya F, Young BM, Fidler JL, Johnson CD, Barlow JM, Earnest F 4th: CT enterography as a diagnostic tool in evaluating small bowel disorders: review of clinical experience with over 700 cases. Radiographics 2006;26:641657.

6 Valette PJ, Rioux M, Pilleul F, Saurin JC, Fouque PL, Henry L: Ultrasonography of chronic inflammatory bowel diseases. Eur Radiol 2001;11:1859-1866.

7 Parente F, Maconi G, Bollani S, Anderloni A, Sampietro G, Cristaldi M, Franceschelli N, Bianco R, Taschieri AM, Bianchi Porro G: Bowel ultrasound in assessment of Crohn's disease and detection of related small bowel strictures: a prospective comparative study versus X-ray and intraoperative findings. Gut 2002;50:490-495.
8 Serra C, Menozzi G, Labate AM, Giangregorio F, Gionchetti P, Beltrami M, Robotti D Fornari F, Cammarota T: Ultrasound assessment of vascularization of the thickened terminal ileum wall in Crohn's disease patients using a low-mechanical index real-time scanning technique with a second generation ultrasound contrast agent. Eur J Radiol 2007;62:114-121.

$\checkmark 9$ Migaleddu V, Quaia E, Scano D, Virgilio G: Inflammatory activity in Crohn disease: ultrasound findings. Abdom Imaging 2008;33: 589-597.

10 Drews BH, Barth TF, Hänle MM, Akinli AS Mason RA, Muche R, Thiel R, Pauls S, Klaus J, von Boyen G, Kratzer W: Comparison of sonographically measured bowel wall vas cularity, histology, and disease activity in Crohn's disease. Eur Radiol 2009;19:1379_ 1386.

11 Potthast S, Rieber A, Von Tirpitz C, Wruk D, Adler G, Brambs HJ: Ultrasound and magnetic resonance imaging in Crohn's disease: a comparison. Eur Radiol 2002;12:14161422

12 Pauls S, Gabelmann A, Schmidt SA, Rieber A, Mittrach C, Haenle MM, Brambs HJ Kratzer W: Evaluating bowel wall vascularity in Crohn's disease: a comparison of dynamic MRI and wideband harmonic imaging contrast-enhanced low MI ultrasound. Eur Radiol 2006;16:2410-2417.

13 Pascu M, Roznowski AB, Müller HP, Adler A, Wiedenmann B, Dignass AU: Clinical relevance of transabdominal ultrasonography and magnetic resonance imaging in patients with inflammatory bowel disease of the terminal ileum and large bowel. Inflamm Bowel Dis 2004;10:373-382.
14 Martínez MJ, Ripollés T, Paredes JM, Blanc E, Martí-Bonmatí L: Assessment of the extension and the inflammatory activity in Crohn's disease: comparison of ultrasound and MRI. Abdom Imaging 2009;34:141-148.

15 Schreyer AG, Scheibl K, Heiss P, Feuerbach S, Seitz J, Herfarth H: MR colonography in inflammatory bowel disease. Abdom Imaging 2006;31:302-307.

16 Schober E, Turetschek K, Mostbeck G: Crohn's disease: current imaging approach. Radiologe 1998;1:15-22.

$>17$ Fraquelli M, Colli A, Casazza G, Paggi S, Colucci A, Massironi S, Duca P, Conte D: Role of US in detection of Crohn disease: metaanalysis. Radiology 2005;236:95-101.

18 Negaard A, Sandvik L, Mulahasanovic A, Berstad AE, Klöw NE: Magnetic resonance enteroclysis in the diagnosis of small-intestinal Crohn's disease: diagnostic accuracy and inter- and intra-observer agreement. Acta Radiol 2006;47:1008-1016.

19 Masselli G, Casciani E, Polettini E, Lanciotti S, Bertini L, Gualdi G: Assessment of Crohn's disease in the small bowel: prospective comparison of magnetic resonance enteroclysis with conventional enteroclysis. Eur Radiol 2006;16:2817-2827

20 Miao YM, Koh DM, Amin Z, Healy JC, Chinn RJ, Zeegen R, Westaby D: Ultrasound and magnetic resonance imaging assessment of active bowel segments in Crohn's disease. Clin Radiol 2002;57:913-918.

21 Maconi G, Bollani S, Bianchi Porro G: Ultrasonographic detection of intestinal complications in Crohn's disease. Dig Dis Sci 1996; 41:1643-1648.

-22 Gasche C, Moser G, Turetschek K, Schober E, Moeschl P, Oberhuber G: Transabdominal bowel sonography for the detection of intestinal complications in Crohn's disease. Gut 1999;44:112-117. 